\title{
Comparison of antegrade and retrograde ureterolithotripsy for proximal ureteral stones: a systematic review and meta-analysis
}

\author{
Kazumi Taguchi ${ }^{1}$, Shuzo Hamamoto ${ }^{1}$, Satoshi Osaga ${ }^{2}$, Teruaki Sugino ${ }^{1}$, Rei Unno ${ }^{1}$, Ryosuke Ando ${ }^{1}$, \\ Atsushi Okada ${ }^{1}$, Takahiro Yasui ${ }^{1}$ \\ ${ }^{1}$ Department of Nephro-urology, Nagoya City University Graduate School of Medical Sciences, Nagoya, Japan; ${ }^{2}$ Clinical Research Management \\ Center, Nagoya City University, Nagoya, Japan \\ Contributions: (I) Conception and design: K Taguchi, S Hamamoto, R Ando; (II) Administrative support: T Sugino, R Unno, A Okada, T Yasui; (III) \\ Provision of study materials or patients: S Osaga, A Okada, T Yasui; (IV) Collection and assembly of data: K Taguchi, S Hamamoto, R Ando, T \\ Sugino, R Unno; (V) Data analysis and interpretation: K Taguchi, SHamamoto, S Osaga, R Ando; (VI) Manuscript writing: All authors; (VII) Final \\ approval of manuscript: All authors. \\ Correspondence to: Shuzo Hamamoto, MD, PhD. Assistant Professor, Department of Nephro-urology, Nagoya City University Graduate School of \\ Medical Sciences, 9th-Floor, Kawasumi 1, Mizuho-cho, Mizuho-ku, Nagoya, 467-8601, Japan. Email: hamamo10@med.nagoya-cu.ac.jp.
}

\begin{abstract}
Background: Antegrade percutaneous ureterolithotripsy (URSL) could be a treatment option for large and/or impacted proximal ureteral stones, which are difficult to treat. To review the current approach and treatment outcomes and to compare the efficacy of retrograde and antegrade URSL for large proximal ureteral stones, we evaluated the unique perspectives of both surgical modalities.
\end{abstract}

Methods: This systematic literature review and meta-analysis was performed in July 2020. Articles on human studies and treatment of ureteral stones with URSL were extracted from the PubMed, MEDLINE, Embase, Cochrane Library, Scopus, and the Japan Medical Abstracts Society databases without any language restrictions. The risks of bias for randomized controlled trials (RCTs) and non-randomized controlled trials (non-RCTs) were assessed using the Cochrane risk of tool and the Risk of Bias in Non-randomized Studiesof Interventions tool, respectively.

Results: A total of 10 studies, including seven RCTs and three non-RCTs, were selected for the analysis; 433 and 420 cases underwent retrograde and antegrade URSL, respectively. The stone-free rate (SFR) was significantly higher in antegrade URSL than in retrograde URSL (SFR ratio: 1.17, 95\% CI: 1.12-1.22; $\mathrm{P}<0.001$ ), while the hospital stay was significantly longer in antegrade URSL than in retrograde URSL (standardized mean difference: 2.56, 95\% CI: 0.67-4.46; P=0.008). There were no significant differences in the operation time and the overall complication rate between the two approaches.

Conclusions: Despite the heterogeneity of data and bias limitations, this latest evidence reflects real practice data, which may be useful for decision making.

Keywords: Ureteroscopic lithotripsy; percutaneous nephrolithotomy; antegrade ureteroscopy; impacted stones; proximal ureteral stones

Submitted Sep 25, 2020. Accepted for publication Dec 23, 2020.

doi: $10.21037 /$ tau-20-1296

View this article at: http://dx.doi.org/10.21037/tau-20-1296

\section{Introduction}

Innovations in ureteroscopes and laser technology have expanded the global application of ureteroscopy (URS) $(1,2)$. Based on the recommendations of various guidelines, it has become the first option of treatment for ureteral stones (3).
However, treatment of large proximal ureteral stones by URS remains challenging due to concerns regarding the impaction on the ureteral wall, tortuous ureter, and the narrow lumen of the distal ureter (4). Furthermore, the laser lithotripsy procedure involved could result in the creation 
of residual fragments or ureteral stenosis (5).

In addition to URS, percutaneous nephrolithotomy (PCNL) (a form of minimally invasive surgery) has been widely utilized for large renal and proximal ureteral stones (6). Although thought to be less invasive than open or laparoscopic stone removal, surgeons are sometimes reluctant to proceed with PCNL due to complications related to renal access (7). A variety of renal access methods, such as ultrasound guidance (8) and miniaturized tract, possibly with the utilization of a flexible nephro- or ureteroscope (9), could mitigate the major unique complications of PCNL without reducing its efficacy for stone removal. While the European Association of Urology (EAU) (10) and the Urological Association of Asia (UAA) (11) guidelines suggest that antegrade ureterolithotripsy (URSL) may be a good alternative for the treatment of proximal ureteral stones larger than $10 \mathrm{~mm}$, there is still a lack of evidence on the optimal case for the application of antegrade URSL over retrograde URSL in real practice.

To better understand the features of both retrograde and antegrade URSL, we conducted a systematic review and meta-analysis comparing the treatment outcomes between the two surgical modalities in patients with large proximal ureteral stones. The results of the present analysis are the most recently updated and useful for decision-making discussions between the surgical team and patients with large ureteral stones. We present the following article in accordance with the PRISMA reporting checklist (available at http://dx.doi.org/10.21037/tau-20-1296) (12).

\section{Methods}

\section{Search strategy}

We performed a systematic literature review and metaanalysis in July 2020. The PubMed, MEDLINE, Embase, Cochrane Library, Scopus, and the Japan Medical Abstracts Society databases were scanned for the following keywords: ("impacted stone" OR "kidney stone" OR "urinary stone" OR "ureteral stone" OR "renal calculus" OR "urolithiasis") AND ("transurethral" OR "retrograde") AND ("percutaneous" OR "antegrade") AND ("ureteroscope" OR "ureteroscopy" OR "lithotripsy" OR "lithotomy"). There were no restrictions on the language and publication date, and both abstracts and full text articles were scanned in order to mitigate selection bias.

\section{Inclusion and exclusion criteria and study outcomes}

The inclusion criteria were as follows: (I) studies conducted on patients with ureteral stones, including ureteropelvic stones, (II) comparative studies on retrograde and antegrade URSL, and (III) studies with outcomes including at least two of the following: stone-free rate (SFR), operation time, hospitalization days, overall complication rate, and postoperative hydronephrosis/fever/ureteral injuries. The exclusion criteria were as follows: (I) reviews, (II) letters, (III) commentaries, (IV) case reports, and (V) studies containing patients with congenital abnormalities or solitary kidneys.

The primary outcome measure was SFR, and the secondary outcome measures were the operation time, postoperative hospital stay, and complication rate.

\section{Data extraction}

Two authors (KT and $\mathrm{SH}$ ) independently reviewed the titles and abstracts of the articles identified by the initial search. During primary screening, data were extracted from the articles that met the inclusion criteria. During secondary screening, the full-text articles were assessed for eligibility with respect to the inclusion and exclusion criteria. In case of duplicate publications, the latest published articles were considered. Any disagreements and discrepancies between the two authors were resolved through discussion and consensus with the other authors. The following data were extracted from all eligible full text articles: first author, journal name, year of publication, type of study, methodology, number of patients, patients' baseline characteristics, stone information, type of surgical intervention, and primary and secondary outcomes relevant to the meta-analysis.

\section{Quality assessment}

Three authors independently assessed the risk of bias for each article. The revised Cochrane risk of bias tool for randomized trials (RoB 2) (13) was used to assess the risk of bias in all randomized controlled trials (RCTs). The quality assessment by RoB 2 included the following aspects: bias arising from the randomization process, bias due to deviations from the intended interventions, bias due to missing outcome data, bias in measurement of the outcome, bias in selection of the reported result, and overall risk-ofbias judgment. Each risk of bias was categorized as 'low risk', 'some concerns', and 'high risk'. To assess the risk of bias for all non-RCTs, the risk of bias in non-randomized studiesof interventions (ROBINS-I) tool was utilized (14). This tool assessed the bias due to confounding, bias in selection 
of participants, bias in classification of interventions, bias due to deviations from the intended intervention, bias due to missing data, bias in measurement of outcomes, bias in selection of the reported result, and overall risk-of-bias judgment.

\section{Statistical analysis}

Data analysis was performed using $\mathrm{R}$ version 3.6.3 with the meta and metafor packages. All tests were two-sided with a significance level set at 0.05 . The SFR ratio, standardized mean difference (SMD) of the operation time and hospital stay, and differences in the risk ratio for overall complications between the retrograde and antegrade groups in each study were calculated in advance. Heterogeneities of these measures among the included studies were evaluated by the $I^{2}$-statistic and Cochran's $Q$-test. Regardless of the presence or absence of heterogeneity, a random-effects model with restricted maximum likelihood estimation was applied to synthesize the measures. The Duval-Tweedie's trim-fill procedure was performed for each measure to assess publication bias and to re-estimate the pooled effect by considering the unpublished studies when bias was indicated. As a sensitivity analysis, we also performed an analysis including only RCTs and compared the results with the results of the analysis on all included studies.

\section{Results}

Based on the search strategies and criteria, 666 records were initially identified across all databases. After 70 articles were removed due to duplication, we screened the titles and abstracts of the remaining 596 articles; of these, 550 were excluded due to lack of relevance with our study aim. The remaining 46 full-text articles were screened further to evaluate their eligibility for analysis on the basis of the eligibility criteria and the data quality. Finally, among the 12 studies that were eligible, 10 were included in the metaanalysis. Figure 1 shows the flowchart of the study selection process. These 10 studies included seven RCTs and three non-RCTs that compared retrograde and antegrade URSL and were published between 2006 and 2017. Table 1 summarizes the characteristics of the studies included in the meta-analysis. A total of 433 cases of retrograde URSL and 420 cases of antegrade URSL were eligible for the analyses. All studies included in this systematic review and metaanalysis targeted proximal ureteral stones that were either larger than $15 \mathrm{~mm}$ in size or were impacted stones.

\section{Assessment of risk of bias}

Among the seven RCTs, the overall risk of bias was categorized as either 'low risk' or 'some concerns': Four studies had some concerns regarding bias arising from the randomization process, while two studies had some concerns regarding either bias due to deviations from the intended interventions or bias in the measurement of the outcome. Furthermore, one study had a low-risk of bias. On the contrary, the overall risks of bias in two and one non-RCTs were categorized as 'moderate' and 'serious risk', respectively. Because none of these studies adjusted the patients' baseline characteristics between retrograde and antegrade URSL, they had a moderate or serious risk of bias due to confounding. One study also had a moderate risk of bias in the selection of participants and the reported results. A summary of these risks is shown in Figure 2.

\section{Stone-free rate}

The SFR was reported in all 10 studies. Random-effects analysis identified that the SFR was significantly higher in patients who underwent antegrade URSL than in those who underwent retrograde URSL (SFR ratio: 1.17, 95\% CI: 1.121.22; $\mathrm{P}<0.001)$. No significant heterogeneity was observed among these studies $\left(\mathrm{P}=0.572, \mathrm{I}^{2}=0.1 \%\right)$ (Figure $\left.3 A\right)$.

\section{Operation time}

Statistical baseline data on the operation time for retrograde and antegrade URSL were available for eight included studies. Random-effects analysis revealed no significant differences in the operation time between the two approaches (SMD: 0.63, 95\% CI: $-1.59-2.84 ; \mathrm{P}=0.580$ ). However, there was significant heterogeneity among the eight studies $\left(\mathrm{P}<0.001, \mathrm{I}^{2}=99.2 \%\right)$ (Figure $3 B$ ).

\section{Hospital-stay}

Statistical baseline data on the hospital stay for retrograde and antegrade URSL were available for eight included studies. Random-effects analysis demonstrated that the hospital-stay duration was significantly longer for patients who underwent antegrade URSL than for those who underwent retrograde URSL (SMD: 2.56, $95 \%$ CI: 0.67-4.46; $\mathrm{P}=0.008)$. However, there was significant heterogeneity among the eight studies $\left(\mathrm{P}<0.001, \mathrm{I}^{2}=99.8 \%\right)$ (Figure 3C). 


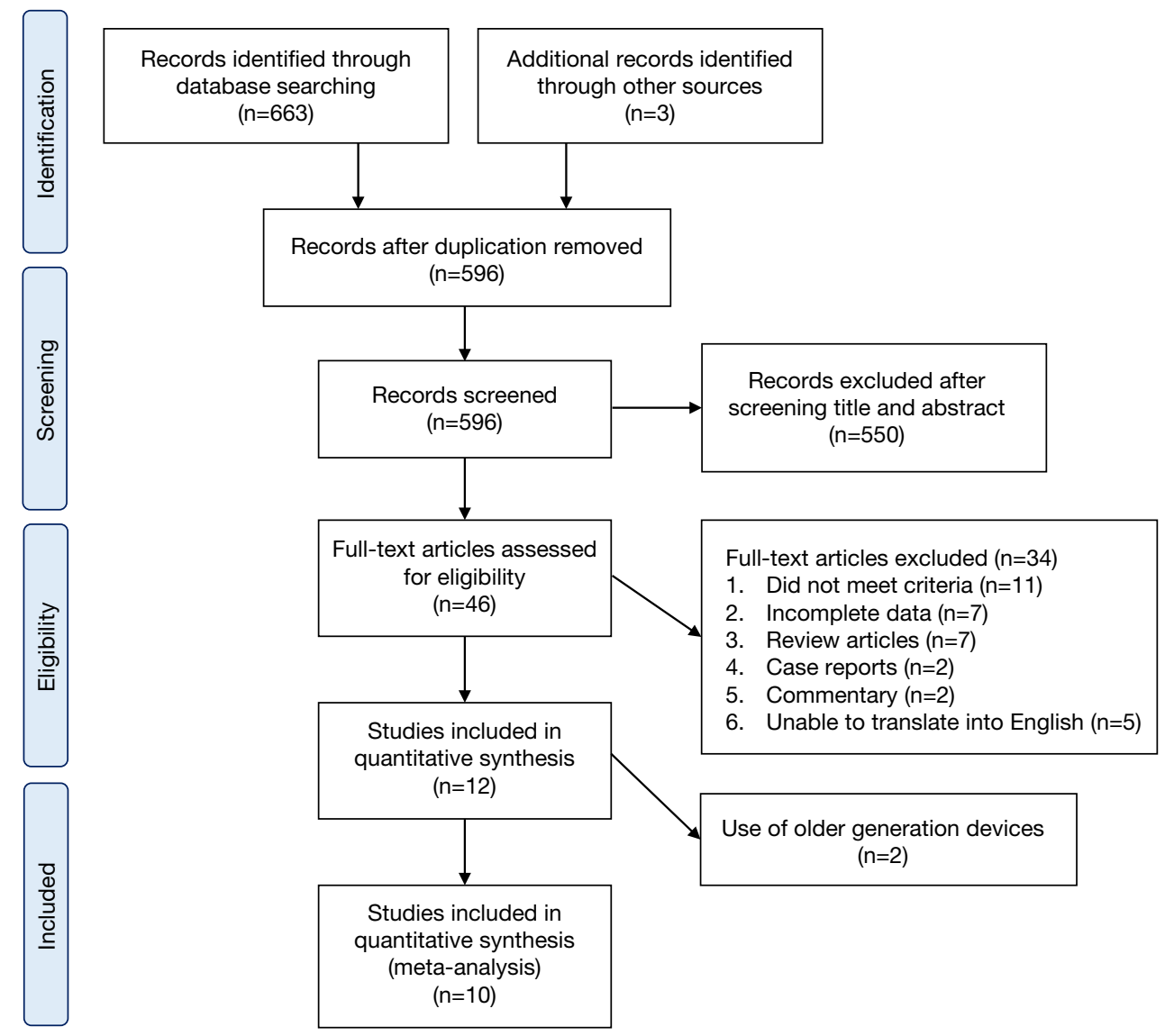

Figure 1 Flowchart depicting the methods used for formulating this systematic literature review in accordance with the PRISMA guidelines.

\section{Overall complications}

Statistical baseline data on the overall complication rates for retrograde and antegrade URSL were available for six included studies. A random-effects model revealed no significant differences in the overall complication rates between the two approaches (SMD: 0.94, 95\% CI: $0.60-1.48 ; \mathrm{P}=0.803)$. No significant heterogeneity was observed among the six studies $\left(\mathrm{P}=0.60, \mathrm{I}^{2}=0 \%\right.$ ) (Figure $3 D$ ). We further performed additional meta-analysis on the incidence of perioperative hemorrhage. While only three studies were suitable for this analysis, a random-effects model revealed that the bleeding rate (BR) was significantly higher in patients who underwent antegrade URSL than in those who underwent retrograde URSL (BR: 3.23, 95\% CI: 1.44-7.25; $\mathrm{P}=0.004)$. However, no significant differences in the transfusion rate (TR) were noted between the two approaches (TR: 5.94, 95\% CI: 0.72-48.46; $\mathrm{P}=0.097$ ) (Figure S1).

\section{Treatment cost}

Only two studies compared the treatment cost between retrograde and antegrade URSL. While we could not perform a meta-analysis for cost comparison due to limited evidence, these articles demonstrated that the cost for antegrade URSL was relatively more than that for retrograde URSL $(\$ 1,785 \pm 274$ vs. $\$ 1,595 \pm 286$ per case in one study and $\$ 1,592 \pm 166$ vs. $\$ 1,107 \pm 81$ per case in another study).

\section{Sensitivity analysis}

Figure 4 shows the results of the meta-analyses with the random-effects models; non-RCTs were excluded from the analyses. The calculated SFR ratio $(1.15,95 \%$ CI: $1.10-1.20)$, SMD of operation time $(0.36,95 \% \mathrm{CI}$ : $-3.30-4.01)$ and hospital stay (3.46, 95\% CI: 0.56-6.36), and risk ratio of complications $(0.75,95 \% \mathrm{CI}: 0.43-1.31)$ 


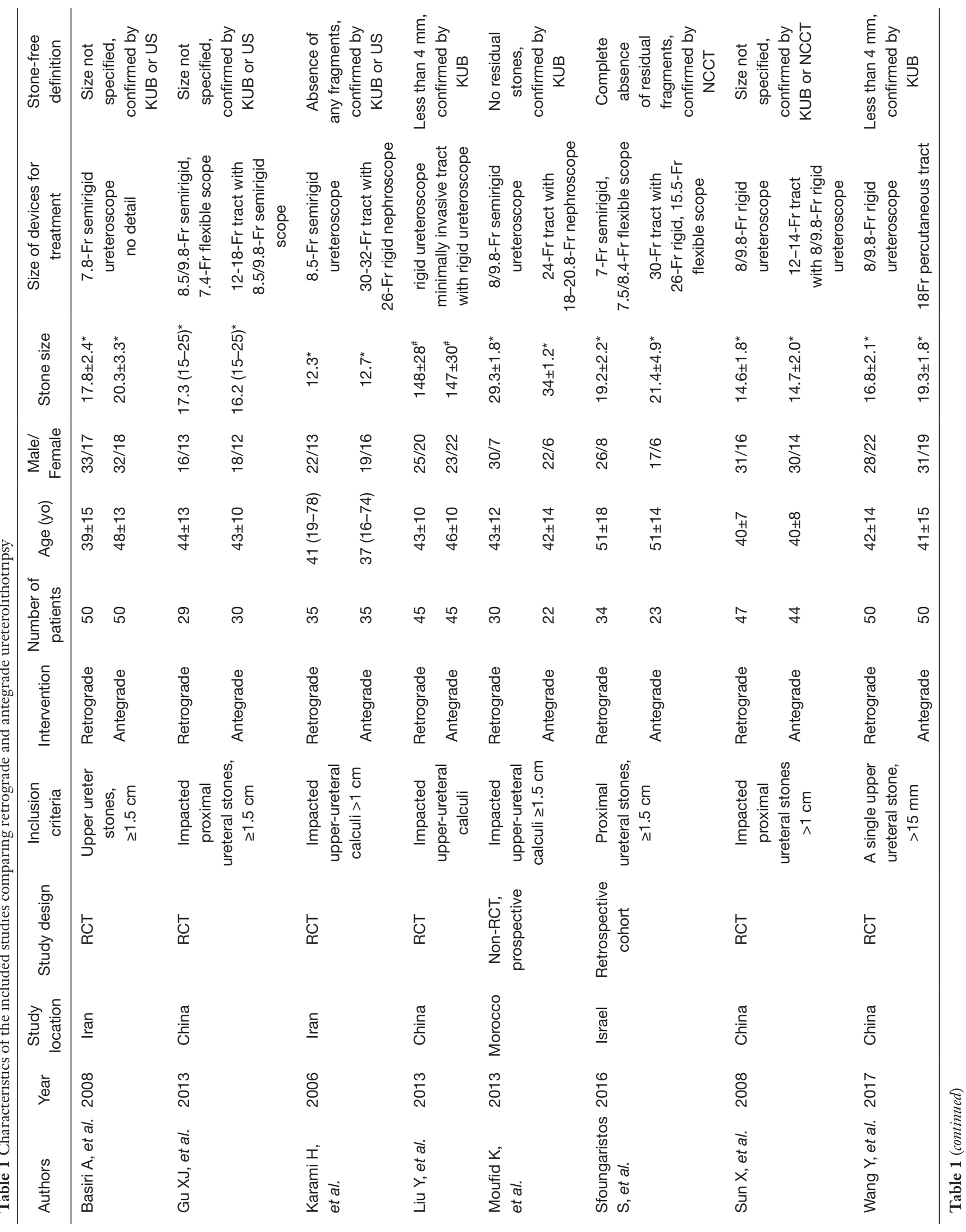




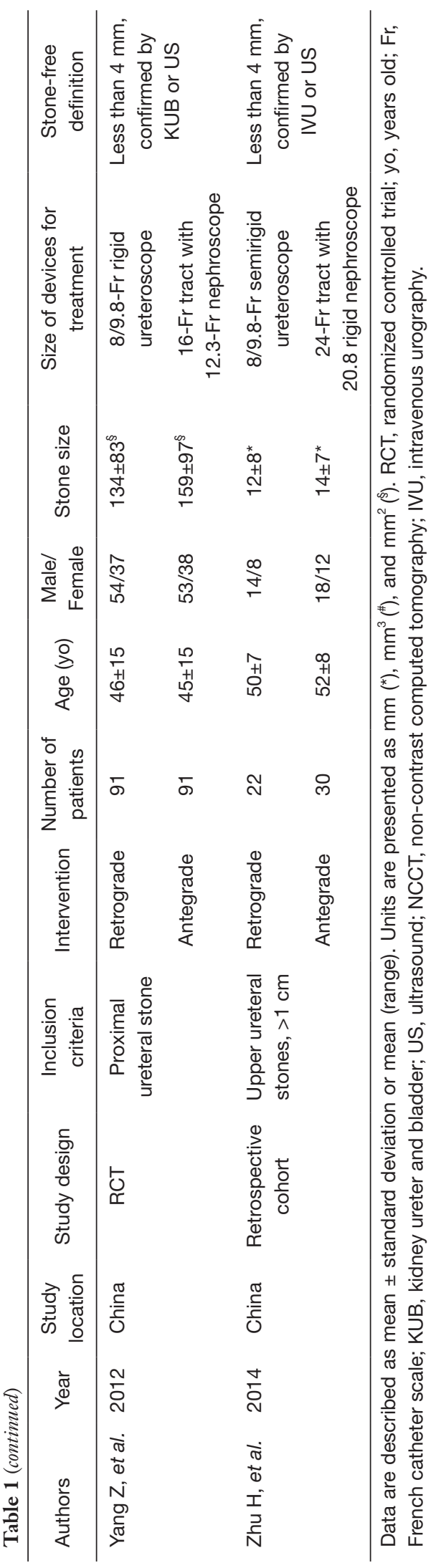

were consistent with the results of all the eligible studies included. Therefore, the results of these meta-analyses were confirmed to be reliable.

\section{Publication bias}

Publication bias was examined using funnel plots. As shown in Figure 5, the funnel plots for SFR and overall complications were asymmetric when three of the estimated missing studies were included. However, the adjusted analyses with filled study populations yielded results comparable to those of the original analyses.

\section{Discussion}

In this systematic review and meta-analysis, we evaluated 10 articles that presented a comparison of retrograde and antegrade URSL for large proximal ureteral stones and were published within the last 15 years (15-24). Our investigations revealed that the SFR was more favorable in antegrade URSL, while the hospital stay was more favorable in retrograde URSL. These findings were consistent with the results of the subgroup analysis performed using only RCTs.

Four modalities are mainly recognized for the treatment of proximal ureteral stones: shockwave lithotripsy (SWL), URSL, PCNL, and laparoscopic ureterolithotomy (LU). Although there are no reports on a direct comparison of the surgical outcomes between these, previous RCTs and/ or systematic reviews and meta-analyses have compared a few of these modalities. A meta-analysis by Cui et al. on 10 comparative studies published between 2004 and 2013 revealed that URSL had a significantly higher initial SFR and lower retreatment rate than SWL for proximal ureteral stones $>10 \mathrm{~mm}$ (25). Wang et al. compared rigid URSL and PCNL (26), and concluded that patients who underwent PCNL were associated with higher SFR rates, but longer operation times and hospital stays, as compared to those who underwent rigid URSL. Furthermore, two metaanalyses from Europe (27) and Brazil (28) on seven and six RCTs, respectively, independently identified that the SFR was higher for LU than for URSL, while the operation and hospitalization times were more favorable in URSL than in LU. Moreover, some studies have compared outcomes between three or more modalities using pooled analyses (29-31). Their data revealed that the SFR after PCNL and LU was significantly higher than that after URSL, but the SFR after URSL was higher than that after SWL. Similar to LU, URSL was also associated with a shorter hospital stay 


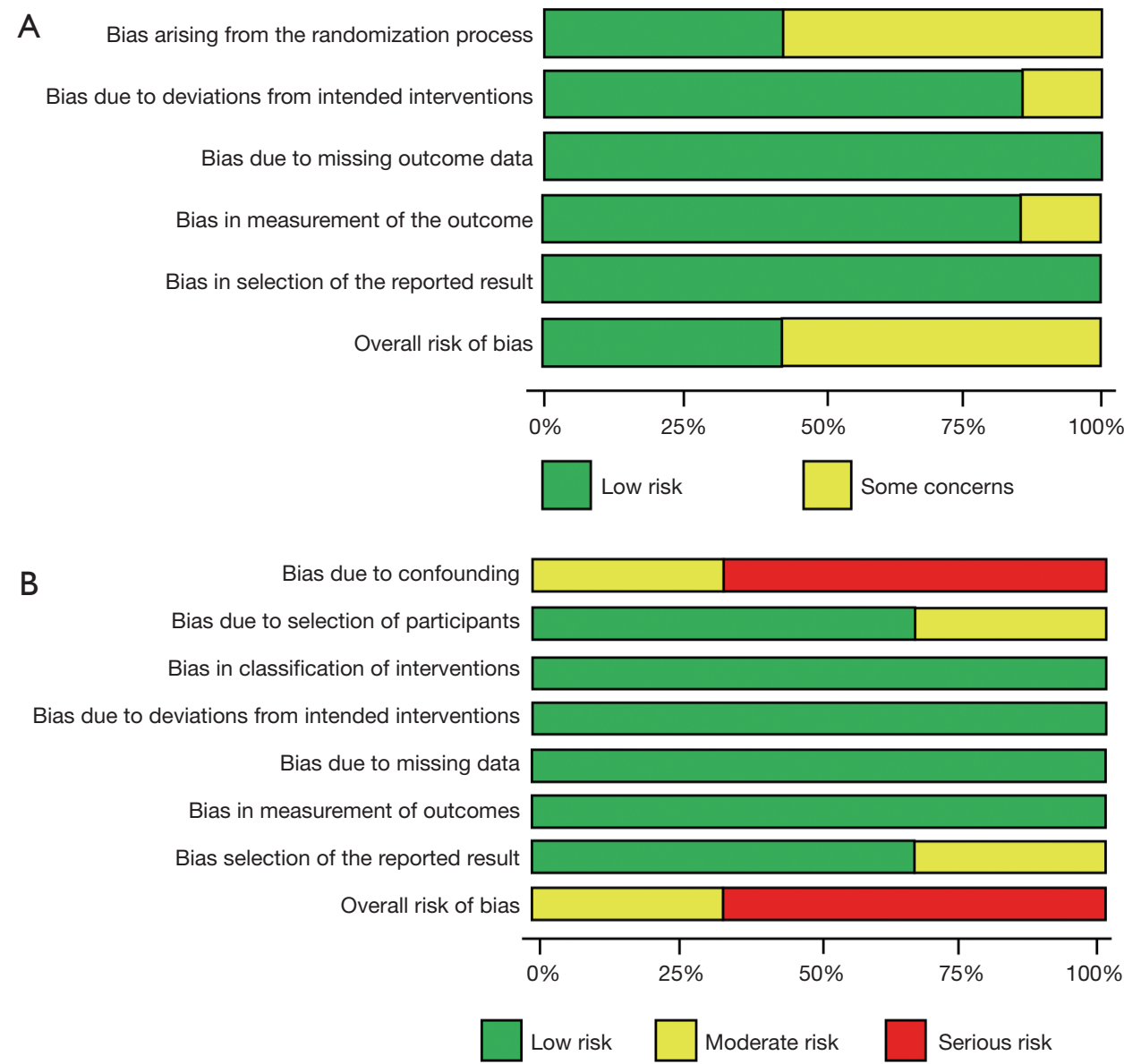

Figure 2 The summary of the risk of bias for randomized controlled trials (A) and non-randomized controlled trials (B).

as compared to PCNL. Regarding complications, URSL was associated with significantly more incidents of ureteral injury as compared to other modalities, while PCNL was associated with a significantly higher blood TR than URSL. These meta-analyses provide evidence on the perspectives and trends of each treatment modality for proximal ureteral stones; however, considering the unique circumstances of each case, each procedure should be applied following a specific and detailed comparison with other modalities. Therefore, we focused on the retrograde and antegrade approaches of URSL in this study, and eventually obtained results consistent with prior research. While we found that there were no significant differences in the overall complication rates between the two approaches (except for a higher BR in the antegrade approach among a small number of studies), we could not undertake meta-analyses of specific complications due to heterogeneity across the articles eligible for the analyses.
Antegrade URSL with 11-French ureteroscope was first described in 1985 by Gumpinger et al. (32). Since then, development of endourological technologies, including flexible ureteroscopy, ureteral access sheath, and laser technology, has enabled the implementation of antegrade URSL with a flexible ureteroscope (33), thereby expanding the treatment options available for ureteral stones. In this study, we first aimed to include a comparison between retrograde URSL and antegrade flexible URSL; however, only one article by Sfoungaristos et al. reported the use of a flexible scope in an antegrade fashion with the Ho:YAG laser fiber. In one study, which was not detected during the literature search due to a difference between the search terms and the study's key words, Bhat et al. reported that in more than one-third of the antegrade cases, a nephroscope successfully allowed access to the upper ureteric stone, which was retrieved by forceps in a procedure that was faster and more effective as compared to the retrograde 
A $\begin{array}{rr}\text { Antegrade } & \text { Retrograde } \\ \text { Author(s) and Year } & \text { Total \# of SF Total \# of SF }\end{array}$

Basiri A et al., 2008

Gu XJ et al., 2013

Karami $\mathrm{H}$ et al., 2006

Liu $Y$ et al., 2013

Moufid K et al., 2013

Sfoungaristos $S$ et al., 2016

Sun $X$ et al., 2008

Wang $Y$ et al., 2017

Yang Z et al., 2012

Zhu $\mathrm{H}$ et al., 2014

Random effects model $\quad \mathbf{4 2 0}$

Heterogeneity: $I^{2}=0 \%, \tau^{2}<0.0001, p=0.54$
SFR Ratio

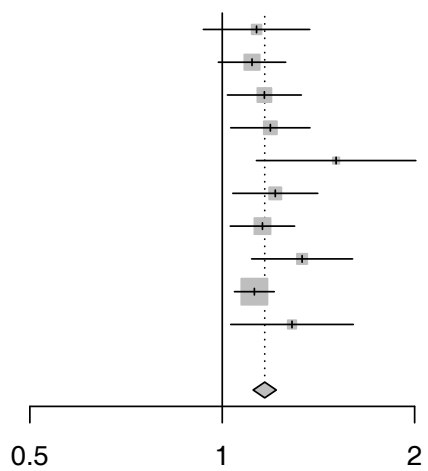

0.5
SFRR $\quad 95 \%-C l$ Weight

$1.13[0.93 ; 1.37] \quad 4.7 \%$

$1.11[0.99 ; 1.26] \quad 11.8 \%$

$1.16[1.02 ; 1.33] \quad 9.8 \%$

$1.19[1.03 ; 1.37] \quad 8.5 \%$

$1.51[1.13 ; 2.01] \quad 2.1 \%$

$1.21[1.04 ; 1.41] \quad 7.4 \%$

$1.16[1.03 ; 1.30] \quad 12.9 \%$

$1.33[1.11 ; 1.60] \quad 5.3 \%$

$1.12[1.04 ; 1.21] \quad 33.8 \%$

$1.29[1.03 ; 1.60] \quad 3.6 \%$

$1.17[1.12 ; 1.22] 100.0 \%$
B

Author(s) and Year

Basiri A et al., 2008

Liu Y et al., 2013

Moufid K et al., 2013

Sfoungaristos $S$ et al., 2016

Sun $X$ et al., 2008

Wang $Y$ et al., 2017

Yang Z et al., 2012

Zhu H et al., 2014

Random effects model $\quad 355$

Heterogeneity: $I^{2}=99 \%, \tau^{2}=10.1029, p<0.01$

Antegrade Retrograde Total Mean SD Total Mean SD
SMD of Operation Time

$\begin{array}{rrrrrr}50 & 93.6 & 28.9 & 50 & 42.7 & 17.9 \\ 45 & 53.8 & 19.2 & 45 & 60.1 & 18.7 \\ 22 & 66.0 & 21.7 & 30 & 52.0 & 17.3 \\ 23 & 63.2 & 12.0 & 34 & 35.3 & 16.0 \\ 44 & 75.4 & 11.8 & 44 & 30.6 & 7.8 \\ 50 & 125.6 & 41.2 & 50 & 55.7 & 23.9 \\ 91 & 27.4 & 2.3 & 91 & 45.2 & 3.1 \\ 30 & 62.2 & 18.3 & 22 & 51.6 & 15.7\end{array}$

366

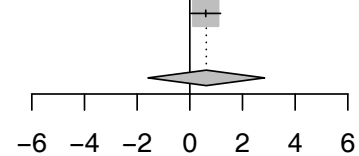

SMD

$2.10 \quad[1.61 ; 2.59] \quad 12.5 \%$

$-0.33[-0.75 ; 0.09] \quad 12.6 \%$

$0.72 \quad[0.15 ; 1.29] 12.5 \%$

$1.89 \quad[1.25 ; 2.53] 12.5 \%$

$4.44 \quad[3.65 ; 5.23] 12.4 \%$

$2.06[1.57 ; 2.55] 12.5 \%$

$-6.49[-7.23 ;-5.76] \quad 12.4 \%$

$0.61 \quad[0.04 ; 1.17] 12.5 \%$

$0.63[-1.59 ; 2.84] 100.0 \%$

\section{C}

Author(s) and Year

Basiri A et al., 2008

Gu XJ et al., 2013

Liu Y et al., 2013

Moufid K et al., 2013

Sfoungaristos S et al., 2016

Sun $X$ et al., 2008

Wang $Y$ et al., 2017

Zhu H et al., 2014
Antegrade Retrograde Total Mean SD Total Mean SD

$\begin{array}{llllll}50 & 4.4 & 1.4 & 50 & 0.5 & 0.1\end{array}$

$\begin{array}{llllll}30 & 4.6 & 1.8 & 29 & 1.9 & 1.3\end{array}$

$\begin{array}{llllll}45 & 6.8 & 3.1 & 45 & 5.2 & 0.7\end{array}$

$\begin{array}{llllll}22 & 2.3 & 0.8 & 30 & 1.7 & 0.6\end{array}$

$\begin{array}{llllll}23 & 4.0 & 1.8 & 34 & 1.6 & 1.5\end{array}$

$\begin{array}{llllll}44 & 6.3 & 0.5 & 44 & 2.1 & 0.4\end{array}$

$\begin{array}{llllll}50 & 6.8 & 2.6 & 50 & 2.5 & 1.3\end{array}$

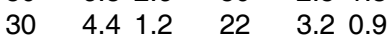

\footnotetext{
Random effects model $\quad 294$

Heterogeneity: $I^{2}=96 \%, \tau^{2}=7.3284, p<0.01$
}

304

SMD of Hospital Stay

SMD $\quad 95 \%-C l$ Weight

$3.87[3.19 ; 4.54] 12.5 \%$

$1.69[1.09 ; 2.29] 12.6 \%$

$0.70[0.28 ; 1.13] \quad 12.7 \%$

$0.86[0.28 ; 1.43] 12.6 \%$

$1.42[0.83 ; 2.02] \quad 12.6 \%$

$9.20[7.74 ; 10.65] \quad 11.9 \%$

$2.08[1.59 ; 2.57] \quad 12.6 \%$

$1.09[0.50 ; 1.68] 12.6 \%$

$2.56[0.67 ; 4.46] 100.0 \%$

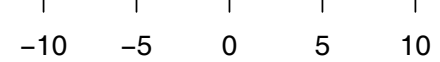

(1)

Author(s) and Year $\begin{array}{r}\text { Antegrade } \quad \text { Retrograde } \\ \text { Total \# of Cx Total \# of Cx }\end{array}$

$\begin{array}{llllr}\text { Liu Y et al., 2013 } & 45 & 4 & 45 & 5 \\ \text { Moufid K et al., 2013 } & 22 & 5 & 30 & 4 \\ \text { Sfoungaristos S et al., 2016 } & 23 & 6 & 34 & 7 \\ \text { Sun X et al., 2008 } & 44 & 1 & 44 & 1 \\ \text { Wang Y et al., 2017 } & 50 & 8 & 50 & 7 \\ \text { Yang Z et al., 2012 } & 91 & 7 & 91 & 14 \\ & & & & \\ \text { Random effects model } & \mathbf{2 7 5} & \mathbf{2 9 4} & \\ \text { Heterogeneity: } I^{2}=0 \%, \tau^{2}=0.0046, p=0.60 & \end{array}$

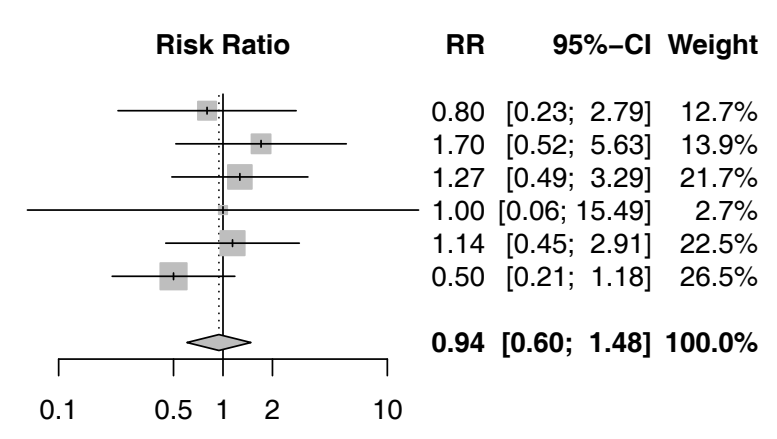

Figure 3 Forest plots and meta-analyses of the stone-free rate (A), operation time (B), hospital stay (C), and overall complications (D) among all eligible studies. \#, number; SF, stone-free; SFR, stone-free rate, SFRR, stone-free rate ratio; CI, confidence interval; SD, standard deviation; SMD, standardized mean difference; $\mathrm{Cx}$, complication; $\mathrm{RR}$, risk ratio. 
A Antegrade
Author(s) and Year
Total \# of SF Total \# of SF

$\begin{array}{lllll}\text { Basiri A et al., 2008 } & 50 & 43 & 50 & 38 \\ \text { Gu XJ et al., 2013 } & 30 & 30 & 29 & 26 \\ \text { Karami H et al., 2006 } & 35 & 35 & 35 & 30 \\ \text { Liu Y et al., 2013 } & 45 & 44 & 45 & 37 \\ \text { Sun X et al., 2008 } & 44 & 44 & 44 & 38 \\ \text { Wang Y et al., 2017 } & 50 & 48 & 50 & 36 \\ \text { Yang Z et al., 2012 } & 91 & 91 & 91 & 81\end{array}$

Random effects model $\mathbf{3 4 5}$

Heterogeneity: $I^{2}=0 \%, \tau^{2}=0, p=0.74$
344

B

Author(s) and Year Total Antegrade Retrograde

Basiri A et al., 2008

Liu Y et al., 2013

Sun $X$ et al., 2008

Wang $Y$ et al., 2017

Yang Z et al., 2012

Random effects model $\mathbf{2 8 0}$

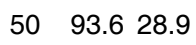

$\begin{array}{lll}45 & 53.8 & 19.2\end{array}$

$\begin{array}{lll}44 & 75.4 & 11.8\end{array}$

$50 \quad 125.6 \quad 41.2$

$\begin{array}{lll}91 & 27.4 & 2.3\end{array}$

Heterogeneity: $I^{2}=99 \%, \tau^{2}=17.3234, p<0.01$

SFR Ratio

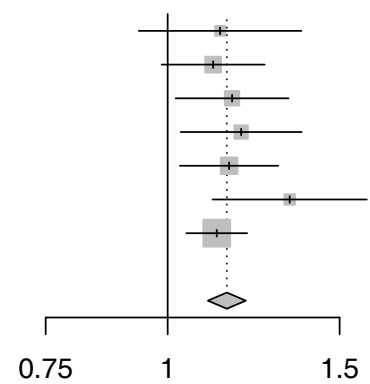

SFRR $\quad 95 \%-C l$ Weight

$1.13[0.93 ; 1.37] \quad 5.4 \%$

$1.11[0.99 ; 1.26] \quad 13.6 \%$

$1.16[1.02 ; 1.33] \quad 11.3 \%$

$1.19[1.03 ; 1.37] \quad 9.8 \%$

$1.16[1.03 ; 1.30] \quad 14.9 \%$

$1.33[1.11 ; 1.60] \quad 6.1 \%$

$1.12[1.04 ; 1.21] \quad 38.9 \%$

$1.15[1.10 ; 1.20] 100.0 \%$

C

\begin{tabular}{|c|c|c|c|c|}
\hline \multirow[b]{2}{*}{ Author(s) and Year } & \multicolumn{2}{|c|}{ Antegrade } & \multicolumn{2}{|c|}{ Retrograde } \\
\hline & Total & lean SD & otal & lean \\
\hline Basiri A et al., 2008 & 50 & 4.41 .4 & 50 & \\
\hline Gu XJ et al., 2013 & 30 & 4.61 .8 & 29 & 1. \\
\hline Liu Y et al., 2013 & 45 & 6.83 .1 & 45 & 5.2 \\
\hline Sun $X$ et al., 2008 & 44 & 6.30 .5 & 44 & 2.1 \\
\hline Wang $Y$ et al., 2017 & 50 & 6.82 .6 & 50 & 2.5 \\
\hline
\end{tabular}

Random effects model $\mathbf{2 1 9}$

218

Heterogeneity: $I^{2}=98 \%, \tau^{2}=10.7804, p<0.01$

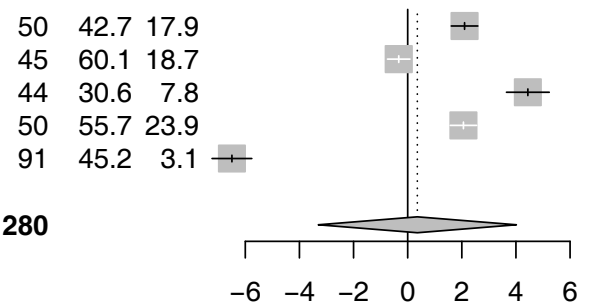

SMD

95\%-Cl Weight

$2.10 \quad[1.61 ; 2.59] \quad 20.0 \%$

$-0.33[-0.75 ; 0.09] \quad 20.1 \%$

$4.44 \quad[3.65 ; 5.23] \quad 19.9 \%$

$2.06 \quad[1.57 ; 2.55] \quad 20.0 \%$

$-6.49[-7.23 ;-5.76] \quad 19.9 \%$

$0.36[-3.30 ; 4.01] 100.0 \%$

SMD of Hospital Stay

SMD

95\%-Cl Weight

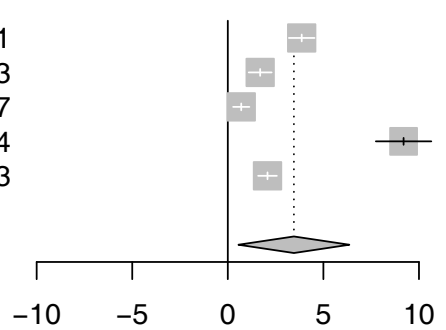

3.87 [3.19; 4.54] 20.1\%

$1.69[1.09 ; 2.29] \quad 20.1 \%$

$0.70[0.28 ; 1.13] \quad 20.2 \%$

$9.20[7.74 ; 10.65] \quad 19.3 \%$

$2.08[1.59 ; 2.57] 20.2 \%$

$3.46[0.56 ; 6.36] 100.0 \%$

D

Author(s) and Year

Antegrade Retrograde

Liu $Y$ et al., 2013

Sun $X$ et al., 2008

Wang $Y$ et al., 2017

Yang $Z$ et al., 2012

Total \# of Cx Total \# of Cx

Random effects model $\mathbf{2 3 0}$

$\begin{array}{lll}45 & 4 & 45 \\ 44 & 1 & 44\end{array}$

$\begin{array}{lll}44 & 1 & 44 \\ 50 & 8 & 50\end{array}$

$\begin{array}{lll}91 & 7 & 91\end{array}$

230

Heterogeneity: $I^{2}=0 \%, \tau^{2}=0, p=0.64$

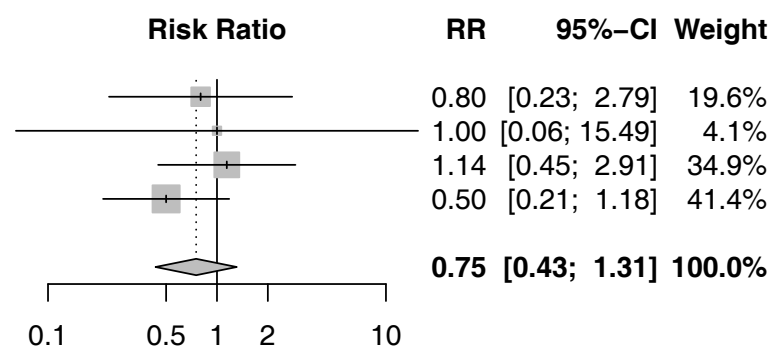

Figure 4 Forest plots and meta-analyses of the stone-free rate (A), operation time (B), hospital stay (C), and overall complications (D) among the randomized controlled studies. \#, number; SF, stone-free; SFR, stone-free rate, SFRR, stone-free rate ratio; CI, confidence interval; SD, standard deviation; SMD, standardized mean difference; Cx, complication; RR, risk ratio.

procedure (34). Surprisingly, all the randomized and nonrandomized comparative studies that we identified set their treatment targets for proximal ureteral stones, and not distal or middle ureteral stones. Antegrade flexible URSL could be feasible for distal and middle ureteral stones; however, the percutaneous antegrade approach has been more suitable for proximal ureteral stones in real-world practice.

As important as the postoperative outcomes of these procedures are, the treatment costs weigh a huge burden on the patients, hospitals, and even the surgeons themselves. 


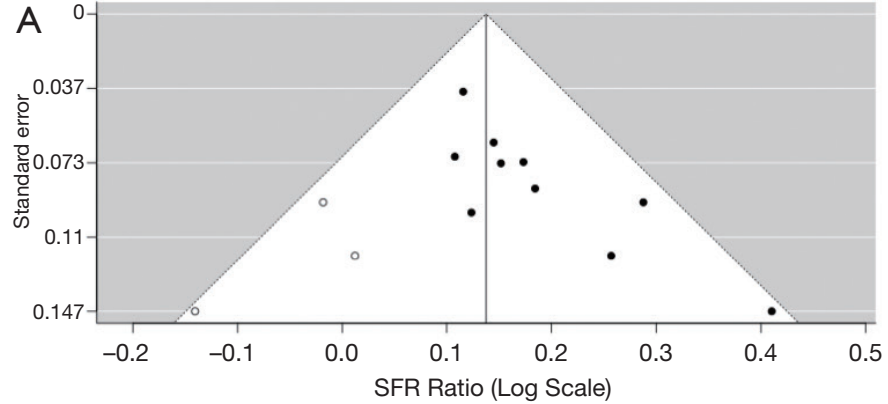

[Result of Bias-Adjusted Analysis]

Estimated number of missing studies: 3

Heterogeneity: $\tau^{2}<0.0001 ; I^{2}=25 \%(P=0.19)$

Test for overall effect: SFRR $=1.14[1.10 ; 1.19]$

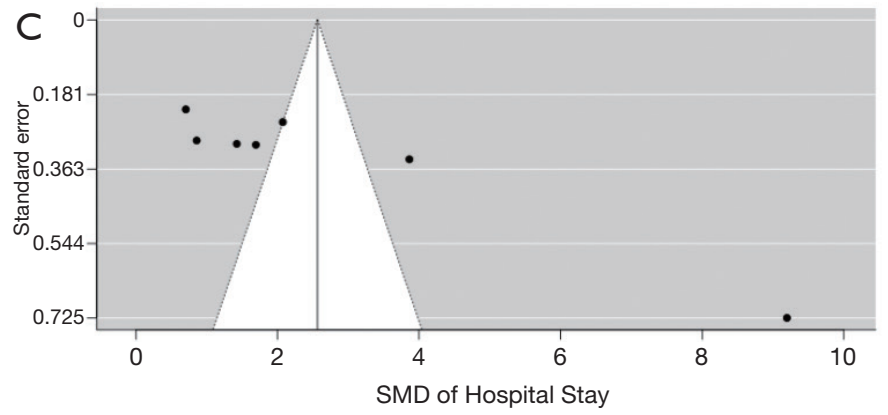

[Result of Bias-Adjusted Analysis] Estimated number of missing studies: 0

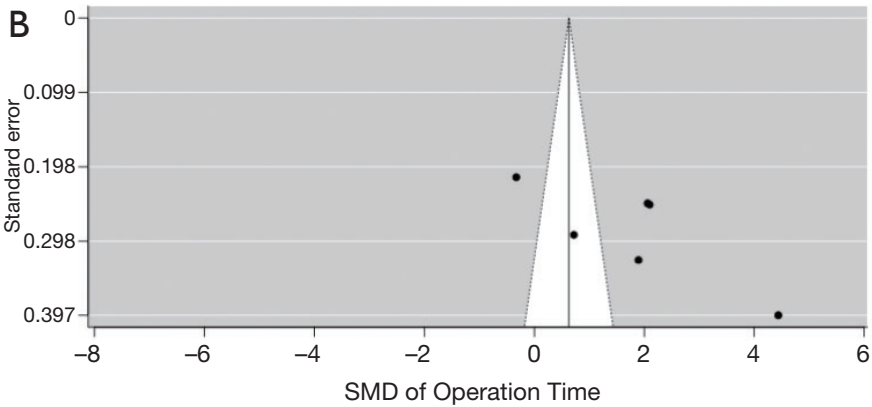

[Result of Bias-Adjusted Analysis]

Estimated number of missing studies: 0

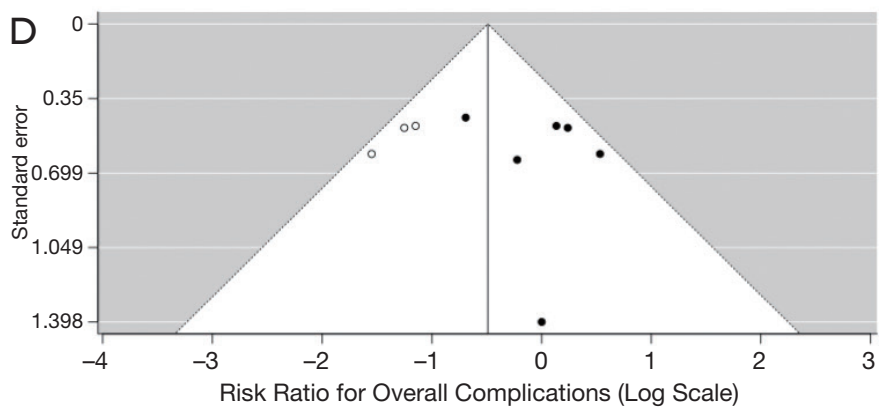

[Result of Bias-Adjusted Analysis]

Estimated number of missing studies: 3

Heterogeneity: $\tau^{2}<0.269 ; l^{2}=45 \%(P=0.07)$

Test for overall effect: $R R=0.61[0.37 ; 1.01]$

Figure 5 Funnel plots of the stone-free rate (A), operation time (B), hospital stay (C), and overall complications (D). SFR, stone-free rate, SFRR, stone-free rate ratio; SMD, standardized mean difference; RR, risk ratio.

Bayne and Chi assessed the cost-effectiveness of the surgical modalities for kidney stones, and suggested that SWL tends to cost the lowest for a single session; however, considering a better SFR, flexible URSL could be less costly than SWL (35). They also presented a perspective of the cost for PCNL, depending on who (urologists or radiologists) obtained renal access, the type of guidance for renal access, and the tract size. However, the cost-effectiveness of LU has not been well-evaluated in recent studies. While we could not provide a cost comparison between retrograde and antegrade URSL due to a limited number of studies that calculated the costs, two studies found that antegrade URSL was a few hundred dollars more expensive than retrograde URSL $(19,23)$. Prior studies compared the costs between different surgical modalities for proximal ureteral stones. Retrograde URSL is less likely to be more costly than PCNL, considering the different use of devices $(29,30)$. Another study from the UK reported that the mean overall cost of treatment of ureteral stones was more favorable for URSL (\$2,801 per case) than for SWL $(\$ 3,627$ per case) (36). Based on this prior evidence, retrograde URSL may be more favorable over other treatment modalities in terms of cost-effectiveness.

Another debatable topic that should be considered prior to treatment is the effect of the modality on renal function. Because all of the studies collected for the present metaanalysis intended to evaluate the surgical outcomes related to stone clearance and major complications, none provided data on the pre- and postoperative renal function. However, Reeves et al. conducted a systematic review of the renal function after URSL and PCNL, and reported that the preand post-surgery renal function did not differ significantly for both treatment modalities; most of the included studies evaluated the renal function using blood parameters, including the creatine level and the estimated glomerular filtration rate (37). Their findings indicated an association between poor preoperative renal function and postoperative renal function deterioration. Similar results were obtained by a systematic review from the EAU Young Academic Urologists and the Uro-Technology Group, indicating 
that the endourological management of stones in a solitary kidney using SWL, URSL, or PCNL did not cause a postoperative decline in the renal function (38). Although these studies included treatment for both, renal and ureteral stones with or without obstruction, the procedural influence for proximal ureteral stones on the renal function (most likely to be hydronephrosis due to obstruction) could not be predicted. Nevertheless, removing the obstruction by either retrograde or antegrade URSL may potentially ameliorate the renal damage caused by obstructive stones, and the effect on renal function should not necessarily differ between the two modalities.

There are some limitations to this study. The main limitation of this review is the heterogeneity in the procedures across the studies included. The tract size and the type of nephroscope used for antegrade and retrograde URSL varied among the studies. This might influence the accuracy of our results when applied in real practice. Furthermore, most RCTs and non-RCTs included in this study lacked double-blinding principles due to ethical concerns. They also lacked a retrospective adjustment for matching cohorts due to the relatively small number of participants in each arm. The insufficient information reported in each study limited subgroup analyses on the complications, costs, and other surgical outcomes. Finally, while our study identified the latest evidence from the most recent studies, there may be a gap in the methods and technology between these studies and the current trend for ureteral stone treatment.

\section{Conclusions}

Our meta-analysis included seven RCTs and three non-RCTs on the most recent endourological procedures for ureteral stones. Our findings indicated that antegrade URSL was associated with a higher SFR but longer hospital stay than retrograde URSL. No statistical differences in the operation time and overall complication rates were observed between the two approaches. Despite the heterogeneity of data and some insufficient information on mitigating biases, the latest evidence reflects real practice data, which may be useful for decision making. This updated investigation of innovative treatment technologies will support further development of strategies for the endourological treatment of stones.

\section{Acknowledgments}

Funding: This work was supported by the Grants-in-Aid for Scientific Research, Japan Society for the Promotion of Science [19H03791, 19K09735, and 20K21658].

\section{Footnote}

Reporting Checklist: The authors have completed the PRISMA reporting checklist. Available at http://dx.doi. org/10.21037/tau-20-1296

Conflicts of Interest: All authors have completed the ICMJE uniform disclosure form (available at http://dx.doi. org/10.21037/tau-20-1296). The authors have no conflicts of interest to declare.

Ethical Statement: The authors are accountable for all aspects of the work and for ensuring that questions related to the accuracy or integrity of any part of the work are appropriately investigated and resolved.

Open Access Statement: This is an Open Access article distributed in accordance with the Creative Commons Attribution-NonCommercial-NoDerivs 4.0 International License (CC BY-NC-ND 4.0), which permits the noncommercial replication and distribution of the article with the strict proviso that no changes or edits are made and the original work is properly cited (including links to both the formal publication through the relevant DOI and the license). See: https://creativecommons.org/licenses/by-nc-nd/4.0/.

\section{References}

1. Giusti G, Proietti S, Villa L, et al. Current standard technique for modern flexible ureteroscopy: tips and tricks. Eur Urol 2016;70:188-94.

2. Pietropaolo A, Proietti S, Geraghty R, et al. Trends of 'urolithiasis: interventions, simulation, and laser technology' over the last 16 years (2000-2015) as published in the literature: a systematic review from European section of Uro-technology (ESUT). World J Urol 2017;35:1651-8.

3. Pradère B, Doizi S, Proietti S, et al. Evaluation of guidelines for surgical management of urolithiasis. J Urol 2018;199:1267-71.

4. Legemate JD, Wijnstok NJ, Matsuda T, et al. Characteristics and outcomes of ureteroscopic treatment in 2650 patients with impacted ureteral stones. World J Urol 2017;35:1497-506.

5. May PC, Hsi RS, Tran H, et al. The morbidity of 
ureteral strictures in patients with prior ureteroscopic stone surgery: multi-institutional outcomes. J Endourol 2018;32:309-14.

6. Kamphuis GM, Baard J, Westendarp M, et al. Lessons learned from the CROES percutaneous nephrolithotomy global study. World J Urol 2015;33:223-33.

7. Rizvi SAH, Hussain M, Askari SH, et al. Surgical outcomes of percutaneous nephrolithotomy in 3402 patients and results of stone analysis in 1559 patients. BJU Int 2017;120:702-9.

8. Armas-Phan M, Tzou DT, Bayne DB, et al. Ultrasound guidance can be used safely for renal tract dilatation during percutaneous nephrolithotomy. BJU Int 2020;125:284-91.

9. Axelsson TA, Cracco C, Desai M, et al. Consultation on kidney stones, Copenhagen 2019: lithotripsy in percutaneous nephrolithotomy. World J Urol 2020. doi: 10.1007/s00345-020-03383-w.

10. Türk C, Petř́k A, Sarica K, et al. EAU Guidelines on interventional treatment for urolithiasis. Eur Urol 2016;69:475-82.

11. Taguchi K, Cho SY, Ng AC, et al. The Urological Association of Asia clinical guideline for urinary stone disease. Int J Urol 2019;26:688-709.

12. Moher D, Shamseer L, Clarke M, et al. Preferred reporting items for systematic review and meta-analysis protocols (PRISMA-P) 2015 statement. Syst Rev 2015;4:14898.

13. Sterne JAC, Savović J, Page MJ, et al. RoB 2: A revised tool for assessing risk of bias in randomised trials. BMJ 2019;366:14898.

14. Sterne JA, Hernán MA, Reeves BC, et al. ROBINS-I: A tool for assessing risk of bias in non-randomised studies of interventions. BMJ 2016;355:14919.

15. Basiri A, Simforoosh N, Ziaee A, et al. Retrograde, antegrade, and laparoscopic approaches for the management of large, proximal ureteral stones: a randomized clinical trial. J Endourol 2008;22:2677-80.

16. Gu XJ, Lu JL, Xu Y. Treatment of large impacted proximal ureteral stones: randomized comparison of minimally invasive percutaneous antegrade ureterolithotripsy versus retrograde ureterolithotripsy. World J Urol 2013;31:1605-10.

17. Karami H, Arbab AH, Hosseini SJ, et al. Impacted upperureteral calculi $>1 \mathrm{~cm}$ : blind access and totally tubeless percutaneous antegrade removal or retrograde approach? J Endourol 2006;20:616-9.

18. Liu Y, Zhou Z, Xia A, et al. Clinical observation of different minimally invasive surgeries for the treatment of impacted upper ureteral calculi. Pak J Med Sci 2013;29:1358-62.

19. Moufid K, Abbaka N, Touiti D, et al. Large impacted upper ureteral calculi: a comparative study between retrograde ureterolithotripsy and percutaneous antegrade ureterolithotripsy in the modified lateral position. Urol Ann 2013;5:140-6.

20. Sun X, Xia S, Lu J, et al. Treatment of large impacted proximal ureteral stones: randomized comparison of percutaneous antegrade ureterolithotripsy versus retrograde ureterolithotripsy. J Endourol 2008;22:913-7.

21. Sfoungaristos S, Mykoniatis I, Isid A, et al. Retrograde versus antegrade approach for the management of large proximal ureteral stones. Biomed Res Int. 2016;2016:6521461.

22. Wang Y, Zhong B, Yang X, et al. Comparison of the efficacy and safety of URSL, RPLU, and MPCNL for treatment of large upper impacted ureteral stones: a randomized controlled trial. BMC Urol 2017;17:50.

23. Yang Z, Song L, Xie D, et al. Comparative study of outcome in treating upper ureteral impacted stones using minimally invasive percutaneous nephrolithotomy with aid of patented system or transurethral ureteroscopy. Urology 2012;80:1192-7.

24. Zhu H, Ye X, Xiao X, et al. Retrograde, antegrade, and laparoscopic approaches to the management of large upper ureteral stones after shockwave lithotripsy failure: a fouryear retrospective study. J Endourol 2014;28:100-3.

25. Cui X, Ji F, Yan H, et al. Comparison between extracorporeal shock wave lithotripsy and ureteroscopic lithotripsy for treating large proximal ureteral stones: a meta-analysis. Urology 2015;85:748-56.

26. Wang Q, Guo J, Hu H, et al. Rigid ureteroscopic lithotripsy versus percutaneous nephrolithotomy for large proximal ureteral stones: a meta-analysis. PLoS One 2017;12:e0171478.

27. Kallidonis P, Ntasiotis P, Knoll T, et al. Minimally invasive surgical ureterolithotomy versus ureteroscopic lithotripsy for large ureteric stones: a systematic review and metaanalysis of the literature. Eur Urol Focus 2017;3:554-66.

28. Torricelli FC, Monga M, Marchini GS, et al. Semirigid ureteroscopic lithotripsy versus laparoscopic ureterolithotomy for large upper ureteral stones: a metaanalysis of randomized controlled trials. Int Braz J Urol 2016;42:645-54.

29. Lai S, Jiao B, Diao T, et al. Optimal management of large proximal ureteral stones $(>10 \mathrm{~mm})$ : a systematic review and meta-analysis of 12 randomized controlled trials. Int J 
Surg 2020;80:205-17.

30. Deng T, Chen Y, Liu B, et al. Systematic review and cumulative analysis of the managements for proximal impacted ureteral stones. World J Urol 2019;37:1687-701.

31. Wu T, Duan X, Chen S, et al. Ureteroscopic Lithotripsy versus Laparoscopic ureterolithotomy or percutaneous nephrolithotomy in the management of large proximal ureteral stones: a systematic review and meta-analysis. Urol Int 2017;99:308-19.

32. Gumpinger R, Miller K, Fuchs G, et al. Antegrade ureteroscopy for stone removal. Eur Urol 1985;11:199-202.

33. Winter M, Lynch C, Appu S, et al. Access sheath-aided percutaneous antegrade ureteroscopy; a novel approach to the ureter. BJU Int 2011;108:620-2.

34. Bhat A, Singh V, Bhat $M$, et al. Comparison of antegrade percutaneous versus retrograde ureteroscopic lithotripsy for upper ureteric calculus for stone clearance, morbidity,

Cite this article as: Taguchi $\mathrm{K}$, Hamamoto S, Osaga S, Sugino T, Unno R, Ando R, Okada A, Yasui T. Comparison of antegrade and retrograde ureterolithotripsy for proximal ureteral stones: a systematic review and meta-analysis. Transl Androl Urol 2021;10(3):1179-1191. doi: 10.21037/tau-20-1296 and complications. Indian J Urol 2019;35:48-53.

35. Bayne DB, Chi TL. Assessing cost-effectiveness of new technologies in stone management. Urol Clin North Am 2019;46:303-13.

36. Geraghty RM, Jones P, Herrmann TR, et al. Ureteroscopy is more cost effective than shock wave lithotripsy for stone treatment: systematic review and meta-analysis. World J Urol 2018;36:1783-93.

37. Reeves T, Pietropaolo A, Gadzhiev N, et al. Role of endourological procedures (PCNL and URS) on renal function: a systematic review. Curr Urol Rep 2020;21:21.

38. Pietropaolo A, Reeves T, Aboumarzouk O, et al. Endourologic Management (PCNL, URS, SWL) of Stones in Solitary Kidney: A Systematic Review from European Association of Urologists Young Academic Urologists and Uro-Technology Groups. J Endourol 2020;34:7-17. 


\section{Supplementary}

A Antegrade Retrograde Author(s) and Year Total \# of B Total \# of B

$\begin{array}{lrrrr}\text { Gu XJ et al., 2013 } & 30 & 15 & 29 & 5 \\ \text { Sun X et al., 2008 } & 44 & 1 & 44 & 0 \\ \text { Wang Y et al., 2017 } & 50 & 5 & 50 & 0 \\ & & & \\ \text { Random effects model } & \mathbf{1 2 4} & & \mathbf{1 2 3} \\ \text { Heterogeneity: } I^{2}=0 \%, \tau^{2}=0, p=0.68 & \end{array}$

B Author(s) and Year

Gu XJ et al., 2013 Wang $Y$ et al., 2017

Yang Z et al., 2012

Random effects model 171

Heterogeneity: $I^{2}=0 \%, \tau^{2}=0, p=0.88$
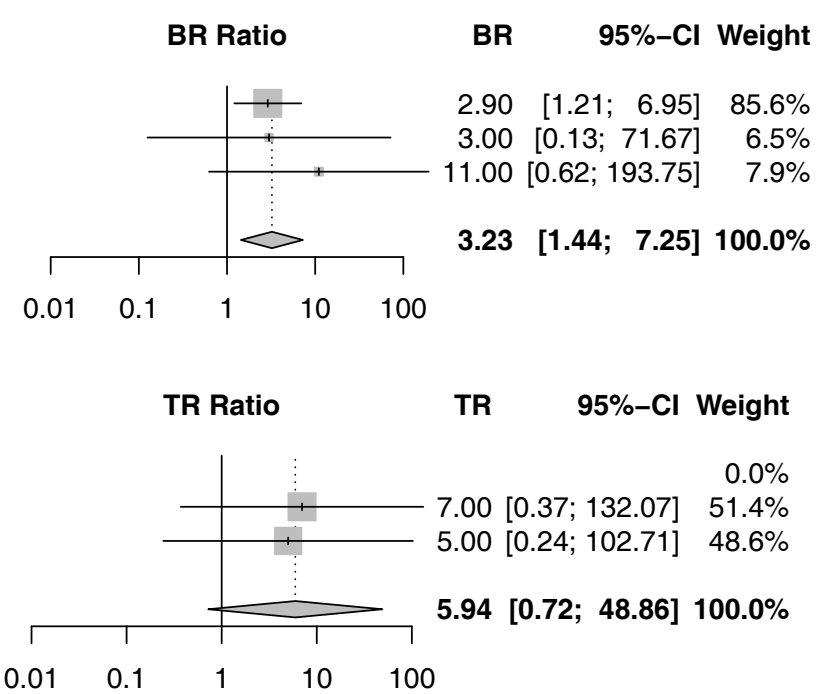

Figure S1 Forest plots and meta-analyses of the bleeding rate (A) and transfusion rate (B) among all eligible studies. \#, number; B, bleeding; $\mathrm{BR}$, bleeding rate; $\mathrm{CI}$, confidence interval; $\mathrm{T}$, transfusion; $\mathrm{TR}$, transfusion rate. 